\title{
Filippo BONINI BARALDI, Tsiganes, musique et empathie
}

Paris, Éditions de la Maison des sciences de l'homme, 2013. (Un DVD-

ROM encarté)

\section{Miriam Rovsing Olsen}

\section{(2) OpenEdition}

Journals

Édition électronique

URL : https://journals.openedition.org/clo/2069

DOI : 10.4000/clo.2069

ISSN : 2266-1816

Éditeur

INALCO

\section{Édition imprimée}

Date de publication : 1 janvier 2013

ISBN : 978-2-85831-217-7

ISSN : 0396-891X

\section{Référence électronique}

Miriam Rovsing Olsen, « Filippo boninI BARALDI, Tsiganes, musique et empathie », Cahiers de littérature orale [En ligne], 73-74 | 2013, mis en ligne le 11 mai 2015, consulté le 01 juillet 2021. URL : http:// journals.openedition.org/clo/2069; DOI : https://doi.org/10.4000/clo.2069

Ce document a été généré automatiquement le 1 juillet 2021.

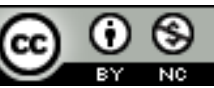

Cahiers de littérature orale est mis à disposition selon les termes de la Licence Creative Commons Attribution - Pas d'Utilisation Commerciale 4.0 International. 


\section{Filippo BONINI BARALDI, Tsiganes, musique et empathie}

Paris, Éditions de la Maison des sciences de l'homme, 2013. (Un DVDROM encarté)

Miriam Rovsing Olsen

1 Basé sur plusieurs années de terrain et sur une connaissance approfondie de plusieurs disciplines (ethnomusicologie, anthropologie, neurosciences), cet ouvrage de Filippo Bonini Baraldi propose une approche originale de la musique d'une communauté de Tsiganes d'un village hongrois en Transylvanie. Le thème retenu de l'émotion musicale paraît ici particulièrement fécond au vu de l'importance que revêtent, dans cette communauté, les manifestations émotionnelles dans de nombreux événements musicaux. L'auteur a choisi de centrer ses recherches sur les «pleurs musicaux ", c'est-à-dire " ces situations où larmes et musique vont de pair » (p. 32). L'émotion musicale se révèle pleinement par l'action et les stratégies des musiciens et dans la dynamique des relations entre les différentes personnes à travers le prisme de l'empathie, concept central de cette recherche qui repose sur un terme local: milă signifiant à la fois « pitié », « compassion » et « empathie ».

2 L'ouvrage est construit en trois parties selon une progression qui amène le lecteur du particulier (l'ethnographie) au général (l'anthropologie) en passant par une étape intermédiaire de comparaison au niveau local. La première partie offre trois situations dans lesquelles surgissent des "pleurs musicaux " grâce aux musiciens : le « service " professionnel (mariages, baptêmes, banquets, bals) où les musiciens (violons, altos à trois cordes dits contră, et contrebasses) sont rémunérés et jouent pour des clients en cherchant à répondre à leurs goûts musicaux (Hongrois, Roumains, Tsiganes) ; les fêtes "spontanées», qui ont lieu dans l'intimité, en famille ou au retour du service professionnel lorsque les musiciens se retrouvent entre eux (dans ce dernier cas, ces fêtes sont aussi qualifiées par Bonino Baraldi de "post-performances»); enfin, les rites funéraires dans lesquels les "proches» du défunt manifestent leur chagrin tout en cherchant à engager, dans leur émotion, les personnes "éloignées » du défunt et à provoquer chez elles la milă. Dans la deuxième partie du livre, l'auteur compare ces 
trois situations et propose un modèle local de l'émotion musicale qui varie en fonction du contexte de performance. Il détermine trois modalités d'émotion musicale: la «fabrication» (service professionnel), le "partage » (funérailles) et "l'expression » (performances musicales intimes), chaque modalité étant définie par douze facteurs de variabilité : 1) le degré de prévisibilité, de préorganisation de la performance musicale ; 2) le statut du musicien et l'enjeu économique ; 3) le public ; 4) la relation de pouvoir entre musiciens et public ; 5) la posture et la maîtrise de soi (fatigue, alcool) ; 6) les relations entre les musiciens ; 7) le choix du répertoire ; 8) le choix des temps de jeu ; 9) le destinataire de la musique ; 10) le protagoniste principal de l'émotion; 11) le lien entre émotion et action musicale; 12) le type d'expérience vécue. En vue de la comparaison, l'auteur choisit d'aborder les invariants des trois situations qui mettent en relation pleurs et musique : un répertoire dit de jale ( « de chagrin »), la relation entre les mélodies et les personnes et une qualité particulière au Tsigane, appelée milos ("généreux ", "sensible», "empathique »), terme dérivé de la milă (voir ci-dessus). Bonini Baraldi parvient de plus à déterminer dans la musique les éléments qui favorisent les pleurs et relèvent d'une " esthétique musicale explicite de la tristesse et du chagrin» (p.34): le rythme non mesuré, le swing (qui se manifeste par une désynchronisation entre mélodie et accompagnement) et des procédés d'ornementation de la mélodie se référant à la « douceur » (dulceaţa). Pour ses analyses, il a eu recours à des outils technologiques sophistiqués, notamment en ce qui concerne la capture du mouvement et sa modélisation (des analyses interactives figurent sur le DVD encarté). La troisième partie développe une théorie anthropologique de l'empathie musicale qui se base sur la théorie d'agency (" agentivité ») d'Alfred Gell. Évoquant cinq "problématiques principales" par rapport à l'empathie musicale (la pertinence du concept ; la nature du référent ; le contexte de performance ; le lien avec les mélodies personnelles; les propriétés formelles des airs de jale), Bonini Baraldi développe sa réflexion à partir d'une importante littérature anthropologique dans laquelle les neurosciences occupent une place centrale. Critiquant les théories courantes de l'émotion musicale, il substitue à celles-ci une approche de l'émotion en termes de « réseaux de relations ». Il établit ainsi une typologie de l'empathie musicale, dont on notera en particulier qu'elle fait éclater les frontières entre personnes et objets, puisque les mélodies sont considérées comme des fragments de personnes défuntes ou absentes dont elles évoquent le souvenir. À ce titre, elles sont parties prenantes d'une intersubjectivité.

De nombreuses fêtes, situées et datées, sont décrites et témoignent de l'intimité de Bonini Baraldi avec les musiciens. Deux d'entre elles, un banquet hongrois et des noces tsiganes, qui figurent sur le DVD encarté, sont détaillées dans leurs trois phases principales (cérémonies officielles; repas et danses; musique à la table des clients), lesquelles sont articulées au sein d'une « suite » selon une progression précise d'airs et de tempo et une interaction sociale de plus en plus étroite entre musiciens et convives. Le beau film d'une heure figurant sur le DVD permet, quant à lui, d'apprécier la large palette sonore des funérailles, en particulier grâce aux lamentations des femmes "proches » du défunt (appelées pleurs «à pleine bouche »). L'une de ces lamentations fait l'objet d'une analyse approfondie à la fois du point de vue de l'acoustique de la voix et des points de vue musicologique et littéraire. De toute évidence, le texte de cent quinze «vers» (dont la moitié fait l'objet également d'une transcription musicale) présente pour l'ethnopoétique un intérêt particulier. En accord avec l'approche générale adoptée pour saisir l'émotion musicale, ce texte poétique est envisagé en 
termes de stratégies et de relations sociales. Ainsi, ce chant d'une jeune femme à sa belle-mère défunte apparaît comme une contribution à la construction de la parenté des proches de la défunte. Par les nombreuses personnes sollicitées, destinataires ou locuteurs et leurs multiples relations de milă (évoquées par un "discours direct»), le texte prend tout son sens. Lors des funérailles, les pleureuses se succèdent et contribuent chacune à la généalogie de la défunte en nommant les personnes qui leur sont chères. À travers l'analyse d'une lamentation funéraire, ce sont donc les mécanismes de construction de la parenté qui sont mis au jour, chaque pleureuse contribuant à construire progressivement un ensemble plus vaste, sur un mode de coénonciation.

Il faut également noter l'intérêt de ce travail pour la connaissance de la mélodie et de son fonctionnement ainsi que les perspectives qui en découlent pour l'ethnopoétique. Bien que sans mots, nous dit Bonini Baraldi, les mélodies instrumentales parlent, à travers cette faculté qu'elles ont de créer des relations de milă comme le font les mots des lamentations. En premier lieu, l'auteur précise l'appartenance de l'air à une "nation", à une "région" ou à un "individu », non pas en fonction de son origine, mais en fonction des destinataires auprès desquels ces mélodies fonctionnent et ont une efficacité émotionnelle. Les airs les plus signifiants, qui mobilisent des souvenirs, sont joués dans les moments intimes et expriment l'empathie musicale avec le client ou avec des personnes aimées du cercle familial, défuntes, souffrantes ou absentes selon les cas; à ce titre, ils provoquent souvent des larmes chez le musicien lui-même (dernière phase des situations de service professionnel ou post-performances). Dans les suites instrumentales des funérailles, les mélodies sont des «images sonores» du défunt et c'est le souvenir de celui-ci, transmis par les mélodies, qui provoque une intensification des pleurs.

5 Il faut souligner le style d'écriture à la fois savant et clair, sans pesanteur, aux formulations souvent heureuses et aux questionnements toujours pertinents, appuyés ou suscités par des extraits d'entretiens avec les Tsiganes (entretiens dont la langue originale figure en note). Cet ouvrage est une contribution majeure à la recherche sur l'émotion musicale ainsi qu'une lecture de bout en bout captivante. 